\title{
Fractional Fourier transform: simulations and experimental results
}

\author{
Yigal Bitran, David Mendlovic, Rainer G. Dorsch, Adolf W. Lohmann, \\ and Haldun M. Ozaktas
}

\begin{abstract}
Recently two optical interpretations of the fractional Fourier transform operator were introduced. We address implementation issues of the fractional-Fourier-transform operation. We show that the original bulk-optics configuration for performing the fractional-Fourier-transform operation [J. Opt. Soc. Am. A 10, $2181(1993)]$ provides a scaled output using a fixed lens. For obtaining a non-scaled output, an asymmetrical setup is suggested and tested. For comparison, computer simulations were performed.

A good agreement between computer simulations and experimental results was obtained.

Key words: Fourier optics, optical information processing, fractional Fourier transforms.
\end{abstract}

Recently the fractional-Fourier-transform (FRT) operator was described in terms of physical optics operations. ${ }^{1-3}$ In this Note we address some experimental aspects of the FRT operator.

The first FRT definition ${ }^{1,2}$ is modeled by the variation of the field during propagation along a quadratic graded-index $($ GRIN $)$ medium by a length proportional to the FRT order $a$. The eigenmodes of quadratic GRIN media are the Hermite-Gaussian (HG) functions, which form an orthogonal and complete basis set. ${ }^{4}$ The $m$ th member of this set is expressed as

$$
\Psi_{m}(x)=H_{m}(\sqrt{2} x / \omega) \exp \left(-x^{2} / \omega^{2}\right),
$$

where $H_{m}$ is a Hermite polynomial of order $m$ and $\omega$ is a constant that is connected with the GRIN-medium parameters. Each function $u(x)$ can be expressed as a linear combination of $\Psi_{m}(x)$, where the coefficient of each HG mode is denoted by $A_{m}$. With the above decomposition the FRT of order $a$ is defined as

$$
\mathscr{f}^{a}[u(x)]=\sum_{m} A_{m} \Psi_{m}(x) \exp \left(i \beta_{m} a L\right),
$$

Y. Bitran and D. Mendlovic are with the Faculty of Engineering, Tel Aviv University, Tel Aviv 69978, Israel. R. G. Dorsch and A. W. Lohmann is with the Angewandte Optik, Erlangen University, Erlangen 8520, Germany. H. M. Ozaktas is with the Department of Electrical Engineering, Bilkent University, Bilkent 06533, Ankara, Turkey.

Received 16 March 1994; revised manuscript received 11 October 1994.

0003-6935/95/081329-04\$06.00/0.

(C) 1995 Optical Society of America. where $L$ is the GRIN length that results in the conventional Fourier transform and $\beta_{m}$ is the propagation constant for each HG mode.

In Ref. 3 the FRT operation is defined alternatively as what happens to the signal $u(x)$ when its Wignerdistribution function (WDF) is rotated by an angle $\phi=a \pi / 2$. Because the WDF of a function can be rotated with bulk optics, Lohmann suggested ${ }^{3}$ use of the bulk-optics system of Fig. 1 for implementing the FRT operator. In his paper, ${ }^{3}$ Lohmann characterized this optical system using two parameters, $Q$ and $R$ :

$$
f=f_{1} / Q, \quad z=f_{1} R,
$$

where $f_{1}$ is an arbitrary fixed length, $f$ is a variable focal length of the lens, and $z$ is the distance between the lens and the input (or the output) plane. For a fractional Fourier transform of order $a, Q$ and $R$ should be chosen as

$$
R=\tan (\phi / 2), \quad Q=\sin (\phi), \quad \phi=a(\pi / 2) .
$$

By analyzing the optical configuration of Fig. 1, one may write

$$
\begin{aligned}
\mathscr{F}^{a}[u(x)]= & C_{1} \int u\left(x_{0}\right) \exp \left(i \pi \frac{x_{0}{ }^{2}+x^{2}}{\lambda f_{1} \tan \phi}\right) \\
& \times \exp \left(-i 2 \pi \frac{x x_{0}}{\lambda f_{1} \sin \phi}\right) \mathrm{d} x_{0},
\end{aligned}
$$

where $\lambda$ is the wavelength and $C_{1}$ is a constant.

Equation (5) defines the FRT for one-dimensional 


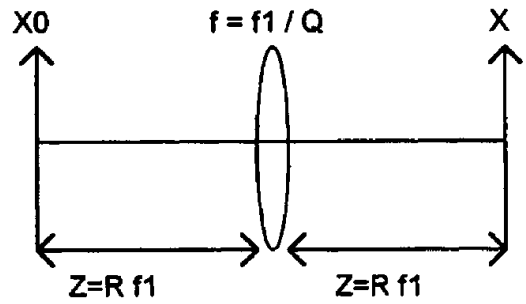

Fig. 1. Setup for performing a two-dimensional fractional Fourier transform according to the WDF definition.

functions. Generalization for two-dimensional functions is straightforward. ${ }^{3}$ This FRT integral definition is fully equivalent to the modal definition given in Eq. (2), as shown in Ref. 5.

Unlike the conventional Fourier-transform operation, which is scale invariant (scaling the input object results in a reciprocal scaling of the output), the generalized FRT is scale variant. ${ }^{1,2}$ Thus if one uses the wrong scaling factor at the input plane, it will be impossible to get the correct FRT output simply by scaling the output plane. In other words it is mandatory to use the correct scaling factor at the input plane.

The influence of changing the scale factor on the HG representation can be found by substitution of the first HG mode $\Psi_{0}(x)$ in Eq. (5). The result is the following relation between $f_{1}$ and the HG parameter $\omega$ :

$$
\omega^{2}=\lambda f_{1} / \pi .
$$

As is mentioned below, computer simulations were done based on the HG representation. Thus the above relation is important to adjust the scale factor of the computer simulations and the bulk-optics experiments.

Equation (5) implies a scale factor of $\left(\lambda f_{1}\right)^{1 / 2}$ for both the input and the output. However, $f_{1}=f \sin [(a \pi) / 2]$ is a function of the FRT order. Thus to keep the scale constant, one needs a zoom lens, which is inconvenient.

To avoid the scale changes when performing the experiments, with the same lens for all fractional orders, we suggest an asymmetrical configuration. Figure 2 shows the optical setup including the two free-space propagation lengths $A f_{1}$ and $B f_{1}$ and the fixed-lens focal length $f=f_{1} / Q$. After some derivation, the output field due to the input $u_{0}\left(x_{0}\right)$ is

$$
\begin{aligned}
u(x)= & \int u_{0}\left(x_{0}\right) \exp \left[\frac { i \pi } { \lambda f _ { 1 } } \left(x_{0}{ }^{2} \frac{1-Q B}{B+A-Q A B}\right.\right. \\
& \left.\left.+x^{2} \frac{1-Q A}{B+A-Q A B}-\frac{2 x x_{0}}{B+A-Q A B}\right)\right] \mathrm{d} x_{0} .
\end{aligned}
$$

We want this output equation to match the FRT definition. By inspecting the coefficients of $x^{2}$ and $x_{0}^{2}$, one notes that the output of the asymmetrical setup is multiplied by a quadratic phase term. Thus

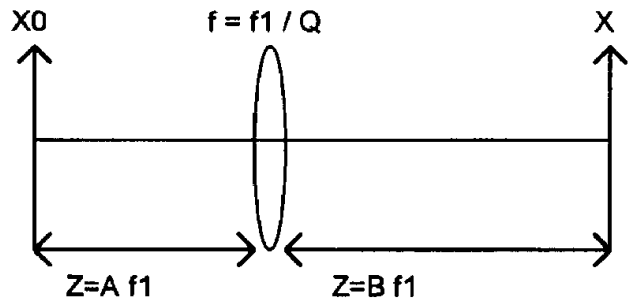

Fig. 2. Setup for performing the fixed-scale asymmetrical FRT.

only the absolute value can be matched to the FRT definition,

$$
|u(x)|=\mid \mathscr{T}^{a}\left[u_{0}\left(x_{0}\right)\right],
$$

by choice of

$$
A=\frac{\sin \phi-(1-\cos \phi) / Q}{\cos \phi}, \quad B=(1-\cos \phi) / Q .
$$

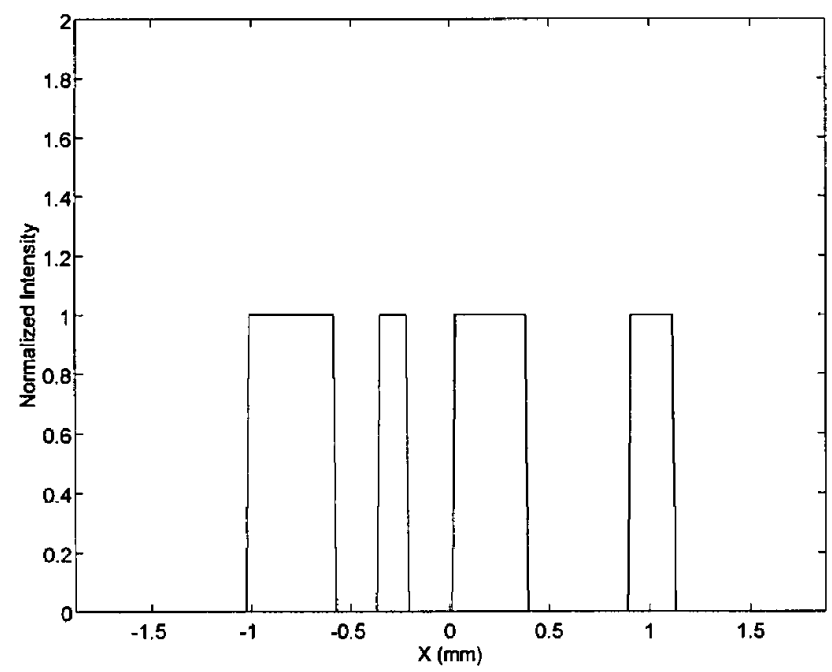

(a)

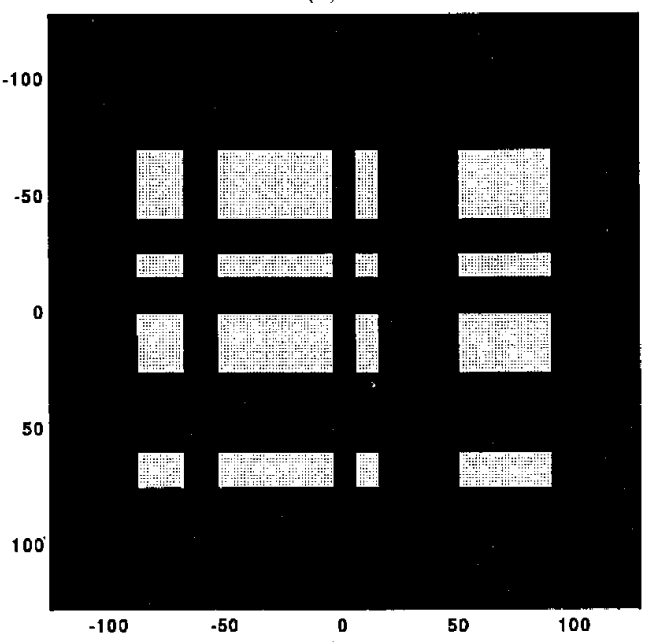

(b)

Fig. 3. Input function (a) cross section and (b) two-dimensional, separable function obtained with a pair of different one-dimensional functions in outer-product form. 


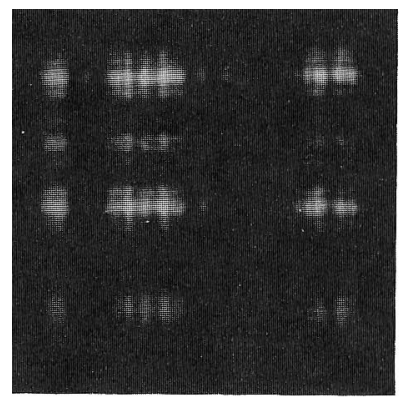

(a)

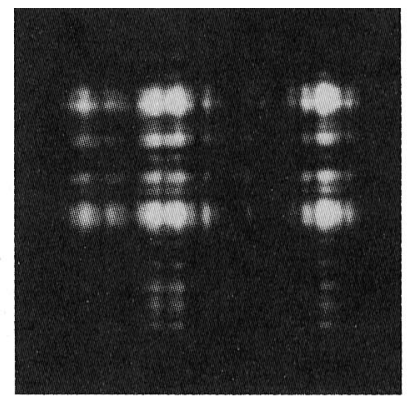

(b)

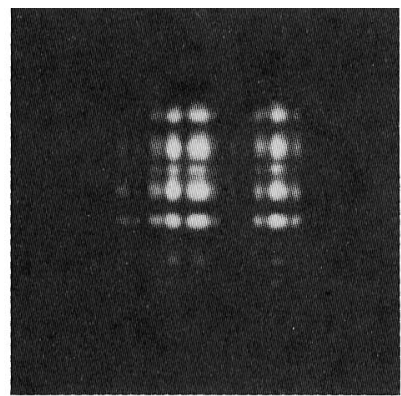

(c)

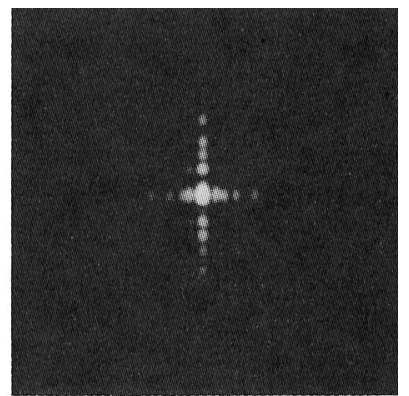

(d)

Fig. 4. FRT experimental result obtained with the optical symmetrical setup of Fig. 1 with the parameters of Table 1 . The FRT order is (a) $a=0.25$, (b) $a=0.5$, (c) $a=0.75$, and (d) $a=1$.

A convenient selection for $Q$ is $Q=1$, which implies $f=f_{1}$.

To acquaint ourselves with the FRT operator, we performed computer simulations. Based on the above two definitions, two approaches were suggested: one is based on the HG definition [Eq. (2)], and the second is based on the WDF definition [Eq. (5)]. Eq. (5) suggests the performance of a Fourier transformation on the input function multiplied by a quadratic phase term (chirp term). Because of the high frequencies necessary to represent truly the chirp term, the resolution necessary to represent the quadratic phase term is much higher than that of the input, requiring a high number of sampling points.

The other FRT computer-implementation approach is based on the HG definition. With the modal notation, ${ }^{2}$ Eq. (2) can be written as

$$
\mathbf{u}_{a}=\mathscr{F}^{a}\left[\mathbf{u}_{0}\right]=\boldsymbol{\Psi} \boldsymbol{\beta}_{a} \boldsymbol{\Psi}^{-1} \mathbf{u}_{0},
$$

where $\beta_{a}$ is a diagonal matrix having $\exp (-i \pi a m / 2)$ as its $m$ th diagonal element, and the $\boldsymbol{\Psi}$ matrix is constructed from the HG functions $\Psi_{m}$ as its rows. Each HG function is normalized by $\left(h_{m}\right)^{1 / 2}$ to ensure that $\boldsymbol{\Psi}$ is orthonormal. We found the $\mathrm{HG}$ approach to be the faster simulation procedure, and thus it was used here for performing the computer experiments.

A MATLAB subroutine was written based on Eq. (10). First, the HG modes $\boldsymbol{\Psi}$ were calculated and stored as a random-access-memory variable. Now each FRT of a vector consists of the product of the input vector by $\boldsymbol{\Psi} \beta_{a} \Psi^{-1} . \quad \boldsymbol{\Psi}^{-1}=\boldsymbol{\Psi}^{T}$, and $\beta_{a}$ is diagonal. Thus the number of operations is $2 N^{2}$ for the two matrixvector multiplications, whereas $\beta_{a}\left(\Psi^{-1} \mathbf{u}_{0}\right)$ is a vectorvector multiplication (only $N$ operations). $\quad \boldsymbol{\Psi}$ is com-

Table 1. Parameters ${ }^{a}$ Used for Performing the Symmetrical Setup Experiments

\begin{tabular}{llllll}
\hline FRT & & & & & \\
Order $a$ & $\phi^{\circ}$ & \multicolumn{1}{c}{$R$} & \multicolumn{1}{c}{$Q$} & $f_{1}(\mathrm{~mm})$ & $Z(\mathrm{~mm})$ \\
\hline 0.25 & 22.5 & 0.199 & 0.383 & 95.75 & 19.05 \\
0.50 & 45 & 0.414 & 0.707 & 176.75 & 73.17 \\
0.75 & 67.5 & 0.668 & 0.924 & 231.0 & 154.31 \\
1 & 90 & 1 & 1 & 250 & 250 \\
\hline
\end{tabular}

${ }^{a}$ These parameters take into account different scale factors for the various FRT orders. puted only once. Consequently each FRT calculation consist mainly of two matrix-vector multiplications, which are performed relatively fast. For example, the number of operations for a 256-element input vector $(N=256)$ is roughly $2 N^{2}=132,000$ operations. The input vector length is always $N$; thus the required number of operations is independent of the fractional order.

The experimental demonstration of the FRT was performed with bulk-optics setups, both for the symmetrical one (Fig. 1) and the asymmetrical one (Fig. 2). Although the GRIN approach could be useful for laboratory experiments, the superiority of the bulkoptics system is apparent because of its much higher SW (space bandwidth product) performance and flexibility. The GRIN approach did serve for the computer simulations.

Figure 3 shows the input function that was used for the simulations and for the experiments. For symmetrical-set-up laboratory experiments a single lens with $f=250 \mathrm{~mm}$ was used. The control of the fractional order was done by a change in the parameter $z$; thus, as mentioned above, the scale of the input and the output depends on the FRT order. The input mask size was $3.75 \mathrm{~mm}$. The distance $z$ to obtain different fractional orders $a$ is given by a combination of Eqs. (3) and (4):

$$
z=f \tan \phi / 2 \sin \phi
$$

where $\phi=a(\pi / 2)$.

Figures $4(\mathrm{a})-4(\mathrm{~d})$ show the experimental results of the intensity distribution for fractional orders a equal to $0.25,0.5,0.75$, and 1 , respectively. The optical setup of Fig. 1 was used with the parameters of Table 1. A fixed lens was used. Thus the various order experiments were done with a different scale factor of

Table 2. Parameters ${ }^{a}$ Used for Performing the Asymmetrical Setup Experiments

\begin{tabular}{clll}
\hline FRT Order $a$ & \multicolumn{1}{c}{$\phi^{\circ}$} & $A f_{1}(\mathrm{~mm})$ & $B f_{1}(\mathrm{~mm})$ \\
\hline 0.25 & 22.5 & 83.0 & 19.0 \\
0.50 & 45 & 146.5 & 73.25 \\
0.75 & 67.5 & 200.25 & 154.25 \\
1 & 90 & 250 & 250 \\
\hline
\end{tabular}

${ }^{a} f$ is $250 \mathrm{~mm}$. 


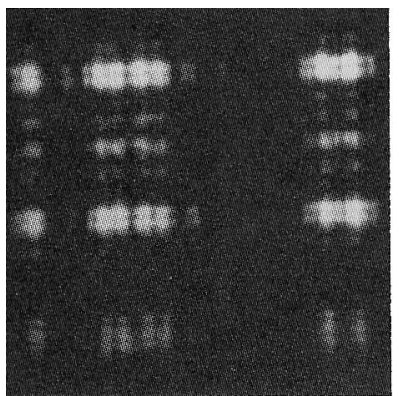

(a)

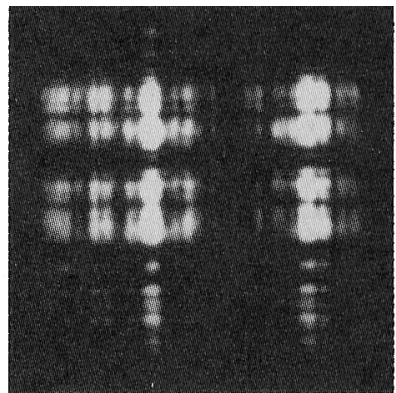

(b)

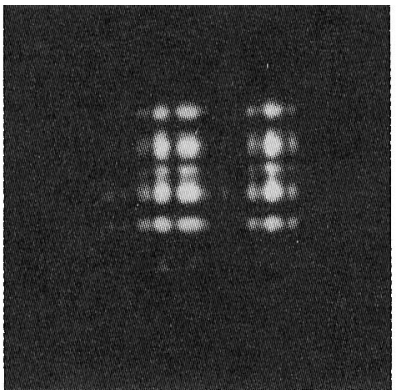

(c)

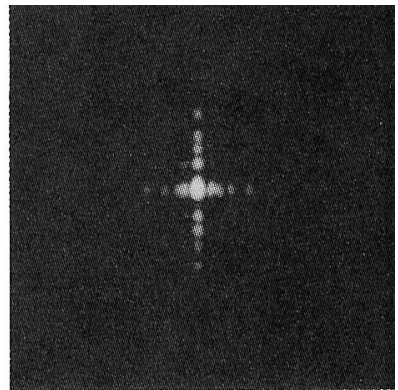

(d)

Fig. 5. FRT experimental result obtained with the asymmetrical optical setup of Fig. 2 with the parameters of Table 2 . The FRT order is (a) $a=0.25$, (b) $a=0.5$, (c) $a=0.75$ and (d) $a=1$.

the input and the output. This was taken into account when the computer simulations were performed, and an excellent agreement of the experimental and computer-simulation results was obtained. For brevity we omitted the computer-simulation plots.

An additional set of experiments was performed in order to explore the asymmetrical configuration. According to Fig. 2, the parameters of the setup were calculated to provide the FRT orders $0.25,0.5,0.75$, and 1 . These parameters are presented in Table $2 ; f$ and $\lambda$ are the same as in the symmetrical experiment. Figure 5 shows the obtained results. The results were compared with the computer-simulation results, and again an excellent agreement was obtained.

To conclude, we have introduced the various possibilities for implementing the fractional-Fouriertransform operation. For computer simulations the GRIN interpretation was used. A bulk-optics optical implementation was suggested in a configuration very similar to the conventional 2- $f$ Fourier-transforming system according to the WDF interpretation. Two optical setups were introduced, a symmetrical and an asymmetrical one. It was shown that, with the symmetrical configuration with a fixed lens, the input and the output objects should be scaled. The asymmetrical setup avoids this scale factor but introduces a quadratic phase distribution to the output plane. Both setups were implemented optically, and experimental results were demonstrated.

\section{References}

1. D. Mendlovic and H. M. Ozaktas, "Fractional Fourier transformations and their optical implementation. Part I," J. Opt. Soc. Am. A 10, 1875-1881 (1993).

2. H. M. Ozaktas and D. Mendlovic, "Fractional Fourier transformations and their optical implementation. Part II," J. Opt. Soc. Am. A 10, 2522-2531 (1993).

3. A. W. Lohmann, "Image rotation, Wigner rotation, and the fractional Fourier transform," J. Opt. Soc. Am. A 10, 2181-2186 (1993).

4. A. Yariv, Optical Electronics, 3rd ed. (Holt, New York, 1985).

5. D. Mendlovic, H. M. Ozaktas, and A. W. Lohmann, "Gradedindex fibers, Wigner distribution functions, and the fractional Fourier transform," Appl. Opt. 33, 6188-6193 (1994). 